\title{
SOLDIER'S RADIO
}

\author{
James R. Schoening \\ Advanced Systems Directorate \\ AMSEL-RD-AS-RA \\ CECOM, Fort Monmouth, NJ 07701 \\ (908) 532-0118
}

and

\section{A. OVERVEW}

The need has been established by the U.S. Army to provide the future soldier with computing and communications capabilities. This will entail providing the individual soldier with a pocket-size computer as well as a communications device for the transmission of both voice and data. These programs are known as Soldier's Computer and Soldier's Radio, and will provide a truly exciting opportunity for the application of the latest in technology.

This paper addresses the Soldier's Radio and many of the unique constraints, issues, factors, and opportunities that will drive the design of this new system.

\section{B. OBJJECTIVE}

Now is the time to influence the decisions and choices being made for this new military communications system. Technical input from industry, government, and academia is desired, and is the objective of this paper.

\section{BACKGROUND}

The Soldier's Radio (SR) is defined as a short range voice/data communications device for the individual soldier. It's primary use is by individuals in squads or small units, but may also be used to interconnect into local and wide area networks. It will be tightly integrated with the Soldier's Computer, which will be a pocket-size processor with a helmet-mounted display, hand held input device, plus a host of other peripheral devices including as Global Positioning System (GPS), digital compass, medical sensors, embedded training, digital maps, etc.

\section{MULTI-MEDIA COMMUNICATIONS}

Combining the capabilities of a computer with a data radio will provide the soldier with "Multimedia Communications". Various forms of communications (voice, voice-mail, e-mail, video, etc.) could be selected depending on factors such as

\author{
Dr. J. Robert Christian \\ $c^{3}$ / Systems Group, Inc. \\ One Executive Drive \\ Tinton Falls, NJ 07701
}

(908) $530-7198$ available battery power, range, security, number of relays, availability of receiving party, nature of the message, and available bandwidth.

\section{E. SCHEDULFAND ACOUISTTION STRATEGY}

Current plans call for the Soldier's Radio to be fielded as a subsystem of The Enhanced Individual Soldier System (TEISS) in 1999. A NonDevelopmental Item (NDI) acquisition strategy is the only feasible approach for various reasons. Time does not permit a full development cycle, funds may not be available for the development of a new radio, and commercial technology is advancing so rapidly that an NDI approach is the only way to keep pace with technology.

\section{F. NETWORKARCHITECTURE}

Network architecture will be a critical consideration in developing the concept for the Soldier's Radio. The operation of the SR can be partitioned into two areas. The architecture required to provide intra-squad (soldier to soldier connectivity), and the architecture required to provide connectivity outside the unit (e.g., to upper and across echelons).

\section{Intra-Squad Connectivity}

The basic radio communications architectures suitable for consideration for the SR intra-squad operations include the Net, Star, and Ring topologies.

The Net Topology, where everyone connects directly to everyone else, is the preferred mode for today's organic Army radio communications, because it is ideally matched to the needs of $a$ military unit. The commander can talk directly to all subordinates at the same time, thus any order given is instantly coordinated. In the broadcast mode the network needs but one frequency. This identifying frequency allows an outside user to contact anyone inside that net. The disadvantage is that an enemy can identify units by the frequency they use.

\subsection{1}


Another consideration of using the net topology concerns broadcast versus private (one-to-one) communications modes. The classic net uses the boradcast mode. Privacy could be added by use of encryption schemes. However, encryption inhibits the instant coordination capabilities of the net. In the case of data transmission, headers can be used to assure that data messages get to their intended destination, but the data transmission protocol must be supportive of the existing classical net communications discipline (listen for the net to be free of traffic before transmitting).

The Star topology, where everyone connects directly to the leader only (Star), is the classical approach utilized in wire networks. Each member is connected to a central point which typically serves as a switching center, connecting members to each other on demand. A radio based star topology would operate in a similar fashion. However, the radio based star topology seems to offer more disadvantages than advantages. The unit leader is the center of the star and becomes the switching center. Only one connection can be activated at any one time. This is a cellular radio technique. Given the radio network is a single frequency system the system is very inflexible. The system is also vulnerable to loss of the central point. The star topology should not be considerred as a potential solution to the Soldier's Radio problem.

The ring topology, where everyone connects to only two other members, creating a ring, has one serious flaw. If one SR fails or one connection is broken, the network goes down. It would also waste power by relaying messages many time around the ring, when a direct connection could be made.

\section{External Squad Connectivity}

Connectivity outside the unit can be achieved either by permitting each user to have direct access to other nets or through a central interface point.

In order for each SR to have direct contact with extenal units, the SR must be able to switch to the operating frequency, contain the appropriate protocols, and be in range of the adjacent unit. Operationally the SR must also have permission to enter another network. The integrated system control, e.g., the allocation of frequencies, the control of security keys, the minimization of traffic overload, the establishment of precedence, etc., becomes a large operational task.

The problems of providing individual connectivity of the SR to users outside its own network are substantially reduced if such connectivity is provided through a central point, namely, the unit leader. Doctrinally, the unit leader has command net connectivity with his adjacent units and their echelon commander (they share the same command net). Therefore he has the radio communications hardward/software to receive and transmit the required messages within that command net. If his unit is supplied with SRs, he will also need HW/SW which provides him connectivity with those SRs. Therefore, he will require two radio systems (which can be in the same box), or one that is programmable to operate in either of the two echelons nets.

The benenfits of the unit leader having a two radio net capability are fourfold: a. The unit leaders system can act as a repeater which provides connectivity from a given SR to or across echelons; b. The unit leader has control of the information/data which is leaving and entering his network; c. The architecture utilized within the SR network can be developed independently of the radio systems utilized in higher echelons; $d$. The design of the SR and its network can be optimized operational, technically, and cost effectively, and any technical and operational issues can be addressed almost independent of the impact of the higher echelon radios.

\section{G. INFORMATION SECURITY}

Information security (INFOSEC) is the protection of information against unauthorized disclosure, modification, or destruction. It has three major components: Communications Security (COMSEC) which is used to encrypt information to deny its authorized use; Transmission Security (TRANSEC) which is used to protect radio frequency emissions from jamming or detection; and Computer Security (COMPUSEC) which provides the means to protect information being handled by computers.

The SR would have the responsibility for providing the COMSEC and TRANSEC capabilities for the soldier's system. The Soldier's Computer would contain the required COMPUSEC.

\section{H. OPERATIONAL FREQUENCYBANDS}

Which frequency band should the Soldier's Radio operate in? The choice of frequency band of operation depends on analysis of a host of interrelationships between the various spectrum alternatives and the Army's operational and organizational requirements. Some of these are: propagation, radio density, separation and replication of frequencies and nets, connectivity requirements, throughput, voice/data requirements, compability, range, cost, and NDI availability. 
The lowest frequency bands have the best propagation conditions and the largest ranges, but they also have severe frequency congestion, require larger antennas, and are bandwidth limited. The UHF and microwave frequency bands have larger bandwidth capabilities, require small antennas, but are limited to line-of-sight (LOS) propagation with some reduction in range in forests. However this is also the band receiving much attention in the commercial market where the needs for data networking are driving the development efforts. The Millimeter wave band which provides very high bandwidth capability, has received much interest by the military because of its covert capabilities. Laser communications is being pursued by commercial interests and small efforts in DoD. The potential problems with MMW and optical propagation are foliage penetration, and range limitations. Ultraviolet transmission is in the early stage of R\&D and shows promise of providing short range non-line-of sight covert connectivity through an atmospheric scatter phenomena.

\section{ADDITIONALCONSIDERATIONS}

$A$ host of additional considerations need to be addressed.

\section{Voice and Data Contention}

How do they share the same channel? Should voice override data? Can voice be packetized and interleaved? Should voice be limited to intrasquad communications, with only data being permitted to interconnect with other networks?

\section{Full Duplex}

How and at what cost can this be achieved for intra-squad communications. How can group conversations be achieved?

\section{Position/Location}

Can the Soldier's Radio be used to help determine the relative location of members of a squad. Could directional antennas, signal strength, or signal delay, in conjunction with Global Positioning System (GPS), pedometers, or other devices be used to triangulate a soldier's position in relation to other members of his squad?

\section{Internetworking}

How will the Soldier's Radio interface with wider area networks?

\section{Protocols}

New protocols will certainly need to be developed that will address constraints such as power conservation, rapidly changing Line-Of-Sight, multipath, voice/data contention, etc.

\section{Power Conservation}

The weight and cost of batteries is one of the most factors of this system. What techniques can be used to conserve power?

\section{Retransmission Unit}

Should unattended retransmission units also be fielded? They could extend range, provide gateways into other networks, save battery power for the soldier, and enhance connectivity where Line-OfSight is a problem.

\section{Electronic Counter-Countermeasures (ECCM)}

Choices of ECCM include frequency hopping, spread spectrum, low probability of intercept, etc.

\section{Antenna}

Where should it be placed on the Soldier?

\section{Density of Radio}

What happens when 10,000 soldier from many different units assemble at an airfield and all want to use their radios?

\section{J. CONCLUSION}

The Soldier's Radio is a new and exciting application of communications technology. It holds the potential to revolutionize tactical military doctrine, but only if the latest in technology can effectively be integrated into a system that will be light, affordable, and functional. Now is the time for those with technical expertise in the many areas of communications technology to influence the decisions that are being made on this system. Such input is welcome by the U.S. Army. 